\title{
Electrophoresis of a non-conducting Newtonian drop of low electrical potential normal to a plane
}

\author{
Eric Lee, Shih-Han Lou, Jyh-Ping Hsu* \\ Department of Chemical Engineering, National Taiwan University, Taipei 10617, Taiwan \\ Received 24 November 2005; received in revised form 21 February 2006; accepted 22 February 2006 \\ Available online 28 February 2006
}

\begin{abstract}
The electrophoretic behavior of a non-conducting, Newtonian drop of low surface potential normal to a plane is investigated theoretically under the conditions of weak applied electric field and arbitrary thickness of double layer. The governing equations and the associated boundary conditions expressed in terms of bipolar spherical coordinates are solved by an orthogonal collocation method. In general, the thinner the double layer surrounding a drop and/or the longer its distance from a planar surface, the larger its mobility, and if a drop is sufficiently close to a plane, its mobility may change sign. These results are similar to the case of a rigid particle. The mobility of a drop decreases with the increase in the ratio (viscosity of drop fluid/viscosity of dispersion medium). Under the conditions assumed, a drop can be treated as a rigid sphere if the viscosity ratio exceeds about 100 , and as a bubble if it is smaller than about 0.01 .
\end{abstract}

(C) 2006 Elsevier Ltd. All rights reserved.

Keywords: Electrophoresis; Boundary effect; Drop normal to plane; Non-conducting Newtonian drop

\section{Introduction}

Electrokinetic phenomena, which include electrophoresis, electroosmosis, sedimentation potential, streaming potential, and electric conductivity, play a key role in colloidal and interfacial science. These phenomena are closely related to the charged conditions of a colloidal entity or a surface. Among the electrokinetic phenomena, electrophoresis is not only a powerful analytical tool for the characterization of the surface properties of a colloidal entity but also one of the key techniques in nanotechnology. While electrophoresis is studied extensively in the past decades, most of the relevant results in the literature focused on rigid entities. In contrast, available results for nonrigid dispersions such as emulsions or micro-emulsions, which are important dispersed systems in practice, are much more limited. Solving the electrokinetic equations for a non-rigid dispersion is usually more complicated than solving those for a rigid dispersion since both the flow and the electric fields inside a non-rigid entity need be considered. The electrophoretic

\footnotetext{
* Corresponding author. Tel.: +8862 23637448; fax: +886223623040.

E-mail address: jphsu@ntu.edu.tw (J.-P. Hsu).
}

behaviors of non-rigid particles was investigated for an isolated mercury drop (Craxford et al., 1937; Booth, 1951; Levich, 1962; Levine and O'Brien, 1973), an isolated drop or gas bubble (Baygents and Saville, 1991a,b; Ohshima, 2003), a dispersion of mercury drops (Ohshima et al., 1984; Ohshima, 1997, 1999; Lee et al., 2003a), a dispersion of general non-rigid particles (Kelsall et al., 1996; Lee et al., 2003b), and a dispersion of non-Newtonian drops (Lee et al., 2005a).

One of the important factors in conducting electrophoresis is the presence of a boundary. This factor needs to be considered, for instance, when a particle is sufficiently close to the wall of electrophoresis cell. Another typical example is in electrodeposition where charged particles are driven by an applied electric field toward electrodes. A thorough review of literature reveals that while many attempts have been made in the past to explore the boundary effect on electrophoresis, most of them are focused on rigid entities (e.g., Keh and Anderson, 1985; Ennis and Anderson, 1997). Relevant results on non-rigid entities, although of both fundamental and practical significance, are very limited. The application of capillary electrophoresis in characterization and separation of vesicles and multi-components liquid separation, for instance, all involve this problem (Breyer et al., 2003; Ashok and Joykrishna, 2005). The analysis of the 
electrophoresis of non-rigid particles is more complicated than that of rigid ones because both the electrokinetic equations inside a particle and those outside a particle need to be considered simultaneously. Lee et al. (2002) analyzed the electrophoresis of a non-rigid particle in a spherical cavity. The electrophoresis (Lee et al., 2004) and the sedimentation (Lee et al., 2005b) of a composite particle in a spherical cavity were studied recently. In general, because the particle-dispersion medium interface is not non-slip, the electrophoretic mobility of a non-rigid particle is larger than that of the corresponding rigid particle.

In this study, the boundary effect on the electrophoresis of a non-rigid particle is analyzed by considering the electrophoresis of a non-conducting Newtonian drop of low surface potential normal to a planar surface. An orthogonal collocation method is adopted for the resolution of the governing electrokinetic equations expressed in terms of bipolar spherical coordinates and the associated boundary conditions. The influences of the thickness of double layer, the distance between a drop and a plane, and relative magnitude of the viscosity of a drop on its electrophoretic behavior are examined.

\section{Theory}

Let us consider the electrophoresis problem illustrated in Fig. 1 where an electrolyte-free drop of radius $a$ moves with velocity $\mathbf{U}$ normal to a planar surface as a response to a uniform applied electric field $\mathbf{E}_{\mathbf{z}}$ of strength $E_{z}$ in the $z$-direction. The distance between the center of the drop and the surface is $h$. The dispersion liquid contains $z_{1}: z_{2}$ electrolytes, $z_{1}$ and $z_{2}$ are, respectively, the valences of cations and anions. Let $n_{10}$ and $n_{20}$ be, respectively, the bulk concentrations of cations and anions with $n_{10}=\alpha n_{20}$. The bipolar spherical coordinates $(\xi, \eta, \varphi)$ are adopted where $\eta=0$ and $\eta=\eta_{0}$ represents, respectively, the planar surface and the drop-dispersion liquid interface, and $\infty>\eta>\eta_{0}$ denotes the domain inside the drop. The bipolar coordinates and the Cartesian coordinates $(x, y, z)$ are related by (Happel and Brenner, 1983)

$$
\begin{aligned}
& z=c \frac{\sinh \eta}{\cosh \eta-\cos \xi}, \\
& y=c \frac{\sin \xi}{\cosh \eta-\cos \xi},
\end{aligned}
$$

where $c$ is the focal length, $0 \leqslant \eta<\infty$, and $0 \leqslant \xi \leqslant \pi$. The physical properties of the dispersion liquid and those of the drop fluid are assumed to maintain at constant values, and the shape of a drop remains spherical.

The electrical potential of the system under consideration, $\phi$, can be described by the Poisson equation

$$
\nabla^{2} \phi=-\frac{\rho}{\varepsilon}=-\sum_{j=1}^{2} \frac{z_{j} e n_{j}}{\varepsilon}
$$

where $\rho$ and $\varepsilon$ are, respectively, the space charge density and the permittivity of the dispersion liquid, $e$ is the elementary charge, and $n_{j}$ is the number concentration of ionic species $j$. If the deformation of double layer surrounding a drop is insignificant, the spatial distribution of ions follows the Boltzmann distribution

$n_{j}=n_{j 0} \exp \left(-\frac{z_{j} e \phi}{k_{B} T}\right)$,

where $T$ is the absolute temperature and $k_{B}$ is the Boltzmann constant. For convenience, $\phi$ is decomposed into an equilibrium potential $\phi_{e}$ and a perturbed potential arising from the applied electric field, $\delta \phi$ (Tang et al., 2001).

For the case $\phi_{e}$ is low, it can be shown that in the dispersion liquid

$\nabla^{2} \phi_{e}^{*}=(\kappa a)^{2} \phi_{e}^{*}, \quad \eta_{0} \geqslant \eta \geqslant 0$,

where $\phi_{e}^{*}=\phi_{e} / \zeta_{a}, \zeta_{a}$ is the potential on drop surface and $n_{j}^{*}=n_{j} / n_{10}$. The reciprocal Debye length $\kappa$ and the scaled gradient operator $\nabla^{2}$ are defined, respectively, by

$\kappa=\left[\sum_{j=1}^{2} n_{j 0}\left(e z_{j}\right)^{2} / \varepsilon k_{B} T\right]^{1 / 2}$,

$\nabla^{2}=\frac{x^{2}}{c^{* 2}}\left[\frac{\partial^{2}}{\partial \eta^{2}}+\frac{\partial^{2}}{\partial \xi^{2}}-\frac{\sinh \eta}{x} \frac{\partial}{\partial \eta}+\frac{\cos \xi \cosh \eta-1}{x \sin \xi} \frac{\partial}{\partial \xi}\right]$

with $c^{*}=c / a$ and $x=\cosh \eta-\cos \xi$. Since the drop is electrolyte-free, the corresponding governing equation for $\phi_{e}^{*}$ is

$\nabla^{2} \phi_{e}^{*}=0, \quad \infty>\eta>\eta_{0}$.

Since $\phi^{*}=\phi_{e}^{*}+\delta \phi^{*}$, Eqs. (3) and (5) lead to

$\nabla^{2} \delta \phi^{*}=\nabla^{2} \phi^{*}-\nabla^{2} \phi_{e}^{*}=0$.

Suppose that both the drop liquid and the dispersion medium are Newtonian, and the flow field can be described by the continuity equation and the Navier-Stokes equation in the creeping flow regime,

$\nabla \cdot \mathbf{v}=0$

$\mu \nabla^{2} \mathbf{v}-\nabla p-\rho \nabla \phi=0$

where $p$ and $\mu$ are, respectively, the pressure and the viscosity. Taking curl on both sides of Eq. (11) and substituting Eqs. (4)(13) into the resultant expression yields

$E^{4} \psi^{*^{(e)}}=-(\kappa a)^{2} \frac{x \sin \xi}{c^{*}}\left(\frac{\partial \phi_{e}^{*}}{\partial \xi} \frac{\partial \delta \phi^{*}}{\partial \eta}-\frac{\partial \phi_{e}^{*}}{\partial \eta} \frac{\partial \delta \phi^{*}}{\partial \xi}\right)$,

where $\psi^{*}=\psi /\left(k_{B} T / z_{1} e\right)^{2} \varepsilon a / \mu$ is the scaled stream function, $\psi$ is the stream function, the superscript $e$ denotes the exterior of a drop, $E^{4}=E^{2} E^{2}$, and

$E^{2}=\frac{x^{2}}{c^{* 2}}\left[\frac{\partial^{2}}{\partial \eta^{2}}+\frac{\partial^{2}}{\partial \xi^{2}}+\frac{\sinh \eta}{x} \frac{\partial}{\partial \eta}+\frac{1-\cos \xi \cosh \eta}{x \sin \xi} \frac{\partial}{\partial \xi}\right]$ 


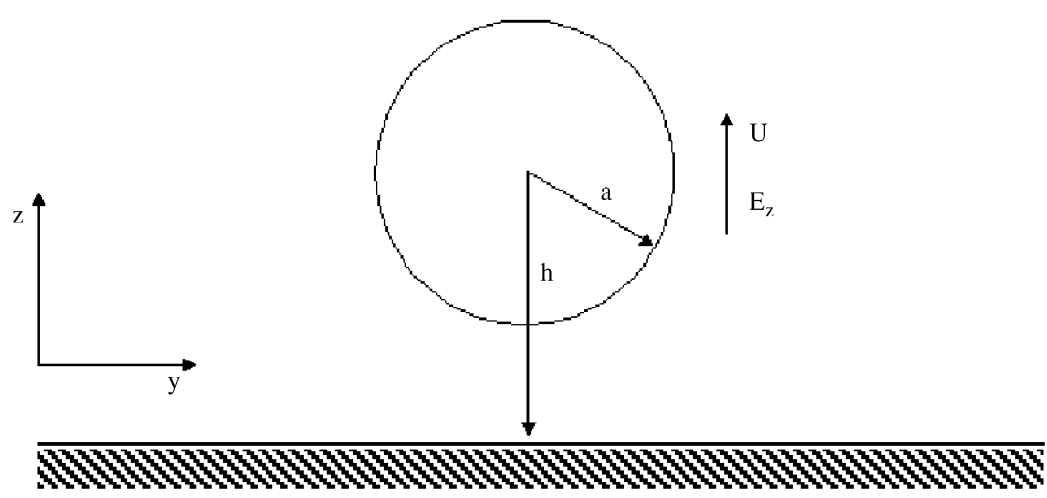

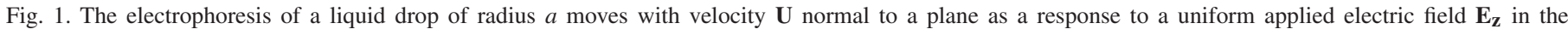
$z$-direction where $h$ is the drop-plane distance.

In terms of $\psi$ and the present bipolar spherical coordinate, the $\eta$ - and the $\xi$-components of the fluid velocity, $v_{\eta}$ and $v_{\xi}$, can be expressed, respectively, as

$v_{\eta}=\frac{x^{2}}{c^{2} \sin \xi} \frac{\partial \psi}{\partial \xi}$,

$v_{\xi}=\frac{-x^{2}}{c^{2} \sin \xi} \frac{\partial \psi}{\partial \eta}$.

Following the same procedure as that employed in the derivation of Eq. (12), it can be shown that for the domain inside a drop

$E^{4} \psi^{*(i)}=0$

where the superscript $i$ denotes the interior of a drop.

We assume that the drop remains spherical and has a uniform equilibrium surface potential. Also, there is no net charge on the plane, and the perturbed potential arising from the applied electric field is normal to the plane. The flow field is assumed to be continuous across the drop-dispersion medium interface, and the planar surface is no-slip. Based on these assumptions, the boundary conditions associated with Eqs. (5), (8), (9), (12), (16) can be expressed as

$\phi_{e}^{*}=1, \quad \eta=\eta_{0}$,

$\phi_{e}^{*}=0, \quad \eta=0$,

$\frac{\partial \phi_{e}^{*}}{\partial \xi}=0, \quad \xi=0$ and $\xi=\pi$,

$\phi_{e}^{*}=0 \quad$ as $\eta \rightarrow \infty$,

$\delta \phi^{*}=-\mathbf{E}_{\mathbf{z}}^{*} \cdot \mathbf{r}^{*}, \quad \eta=0$,

$\frac{\partial \delta \phi^{*}}{\partial \eta}=0, \quad \eta=\eta_{0}$,

$\delta \phi^{*}=0, \quad \eta>\eta_{0}$,

$\psi^{*(e)}=0, \quad \eta=0$,

$\frac{\partial \psi^{*(e)}}{\partial \eta}=0, \quad \eta=0$ $\frac{\partial \psi^{*(e)}}{\partial \eta}-\frac{\partial \psi^{*(i)}}{\partial \eta}=\frac{c^{* 2}}{x^{3}} \sin ^{2} \xi \sinh \eta U^{*}, \quad \eta=\eta_{0}$,

$\psi^{*(e)}=\psi^{*(i)}, \quad \eta=\eta_{0}$

$\frac{\psi^{*(e)}}{r^{2}} \rightarrow 0, \quad \eta=0$ and $\xi=0$

$\psi^{*(e)}=0 \quad$ and $\quad \frac{\partial \psi^{*(e)}}{\partial \xi}=0, \quad \xi=0$ and $\xi=\pi$

$\pi_{\eta \xi}^{*(i)}=\pi_{\eta \xi}^{*(e)}, \quad \eta=\eta_{0}$

In these expressions, $\pi_{\eta \xi}^{*}$ is the dimensionless shear stress on the drop surface, $\mathbf{E}_{\mathbf{z}}^{*}=\mathbf{E}_{\mathbf{z}} /\left(\zeta_{a} / a\right)$ is the scaled electric field, $\mathbf{r}^{*}=(r / a) \mathbf{i}_{\mathbf{r}}$ is the normal vector pointing from the center of a drop to its interface, and $U^{*}=U / U_{E}$, where $U_{E}=\varepsilon \zeta^{2} / \mu_{r} a$.

The solution procedure used by O'Brien and White (1978) is used where an electrophoresis problem is decomposed into two subproblems. In the first subproblem, a drop moves in the absence of the applied electric field, and in the second problem, it is held fixed when the electric field is applied. The mobility of a drop can be evaluated based on the condition that the total force acting on it vanishes at the steady state (O'Brien and White, 1978; Baygents and Saville, 1991a). A pseudo-spectral method based on Chebyshev polynomials (Finlayson, 1980), which is readily applicable to the present problem, is adopted for the resolution of the governing equations and the associated boundary conditions. Convergence test is conduced, and using $30 \times 30$ nodal points inside droplet and $30 \times 45$ outside droplet is found to be sufficiently accurate; Fig. 2 shows the resultant mesh system. The applicability of the present numerical scheme is also justified by comparing the results of Wacholder and Weihs (1972), where a pure hydrodynamic problem was analyzed for the case of a droplet moving normal to a plane, with those evaluated by our method. Tables 1 and 2 summarize the result of comparison. These tables indicate that the performance of the present numerical scheme is satisfactory. 


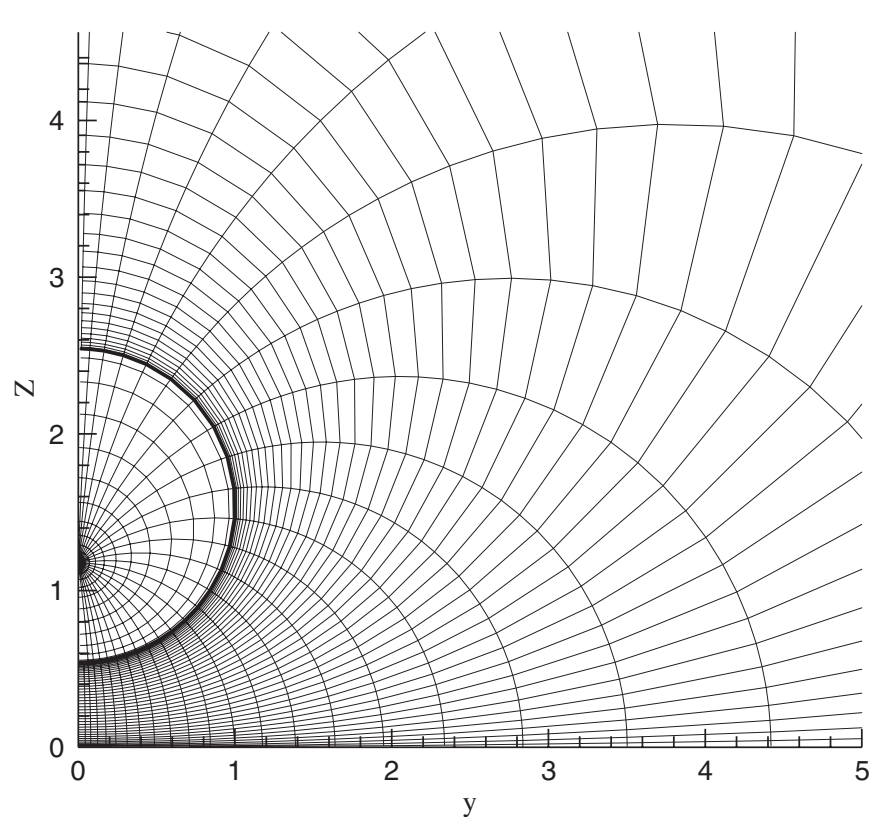

Fig. 2. The system mesh for a liquid drop normal to the plane $\left(\eta_{0}=1.0\right.$; zoom-in).

Table 1

Dimensionless drag on a droplet for the case of a pure hydrodynamic problem for various values of $\eta_{0}$ at $\sigma=1.0$

\begin{tabular}{llll}
\hline$\eta_{0}$ & $\begin{array}{l}\text { Exact analytical } \\
\text { result }\end{array}$ & $\begin{array}{l}\text { Present numerical } \\
\text { result }\end{array}$ & $\begin{array}{l}\text { Percentage } \\
\text { deviation }\end{array}$ \\
\hline 0.5 & 28.9070 & 28.90628 & 0.00249075 \\
1 & 12.14115 & 12.14712 & 0.04921115 \\
1.5 & 8.236236 & 8.238643 & 0.02922451 \\
2 & 6.6335 & 6.686423 & 0.79781413 \\
2.5 & 5.8963 & 5.9568 & 1.0260719 \\
3 & 5.5121 & 5.56999 & 1.05023494 \\
\hline
\end{tabular}

The exact analytical result is reported by Wacholder and Weihs (1972).

Table 2

Dimensionless drag on a rigid plane for the case of a pure hydrodynamic problem for various values of $\sigma$ at $\eta_{0}=1.0$

\begin{tabular}{lccl}
\hline$\sigma$ & $\begin{array}{l}\text { Exact analytical } \\
\text { result }\end{array}$ & $\begin{array}{l}\text { Present numerical } \\
\text { result }\end{array}$ & $\begin{array}{l}\text { Percentage } \\
\text { deviation }\end{array}$ \\
\hline 0 & 8.22184 & 8.68154 & 5.59120586 \\
0.5 & 10.66268 & 10.69016 & 0.25770257 \\
1 & 12.14115 & 12.14712 & 0.04921115 \\
10 & 16.86964 & 16.87936 & 0.05762042 \\
100 & 18.06295 & 18.10583 & 0.23739978 \\
\hline
\end{tabular}

The exact analytical result is reported by Wacholder and Weihs (1972).

\section{Results and discussion}

The influences of the key parameters of the present problem, including viscosity of the drop and boundary effect, on the electrophoretic behavior of a drop are examined through numerical simulation. For illustration, we assume that $P e_{1}=0.01$ and $P e_{2}=0.01$. The contours of the scaled equilibrium electric
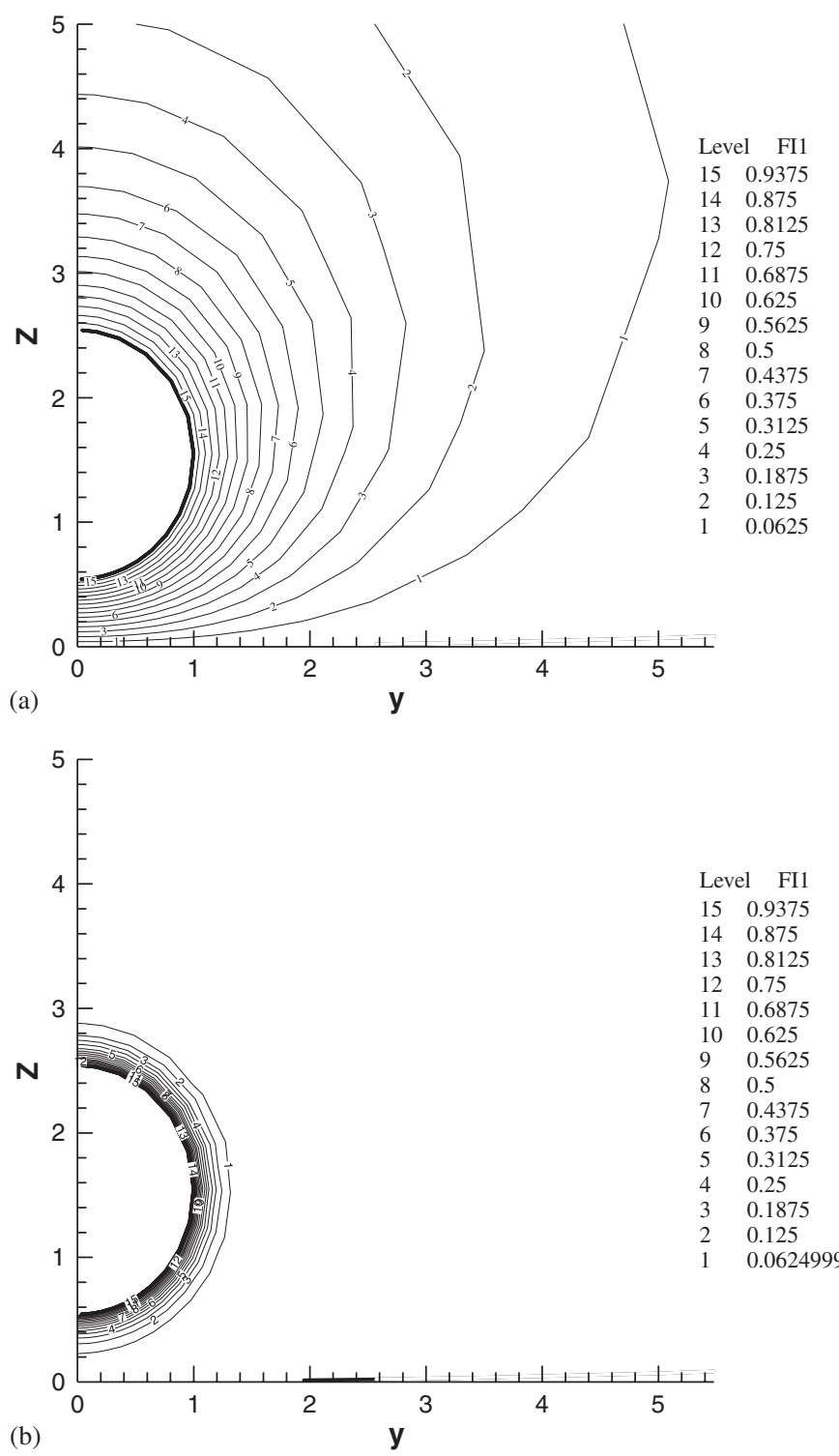

Fig. 3. Contours of scaled equilibrium potential $\phi_{e}^{*}$ for two levels of $\kappa a$ at $\eta_{0}=1.0$, and $\sigma=1.0$ : (a) $\kappa a=0.01$, (b) $\kappa a=7.943$.

potential $\phi_{e}^{*}$ at two levels of $\kappa a$ are presented in Fig. 3, and the corresponding contours of the scaled stream function $\psi^{*}$ are shown in Fig. 4. Fig. 3 indicates that a larger value of $\kappa a$ leads to a narrower distribution in $\phi_{e}^{*}$ and a steeper gradient of $\phi_{e}^{*}$ near the surface of a drop. Since the drop is non-conductive, the latter implies that the larger the value of $\kappa a$, the higher the ion concentration in the solution, the higher the charge density on its surface, and therefore, a greater electric force acting on it, which yields a larger mobility. The flow pattern shown in Fig. 4 justifies this inference. Note that the streamlines are continuous across the drop-dispersion liquid interface, which results from the boundary conditions assumed.

The influence of the nature of a drop, measured by $\sigma=$ $\mu^{(i)} / \mu^{(e)}$, is illustrated in Figs. 5 and 6. For comparison, the corresponding results for the case of a rigid sphere (Ennis and Anderson, 1997; Tang et al., 2001) are also presented in these 


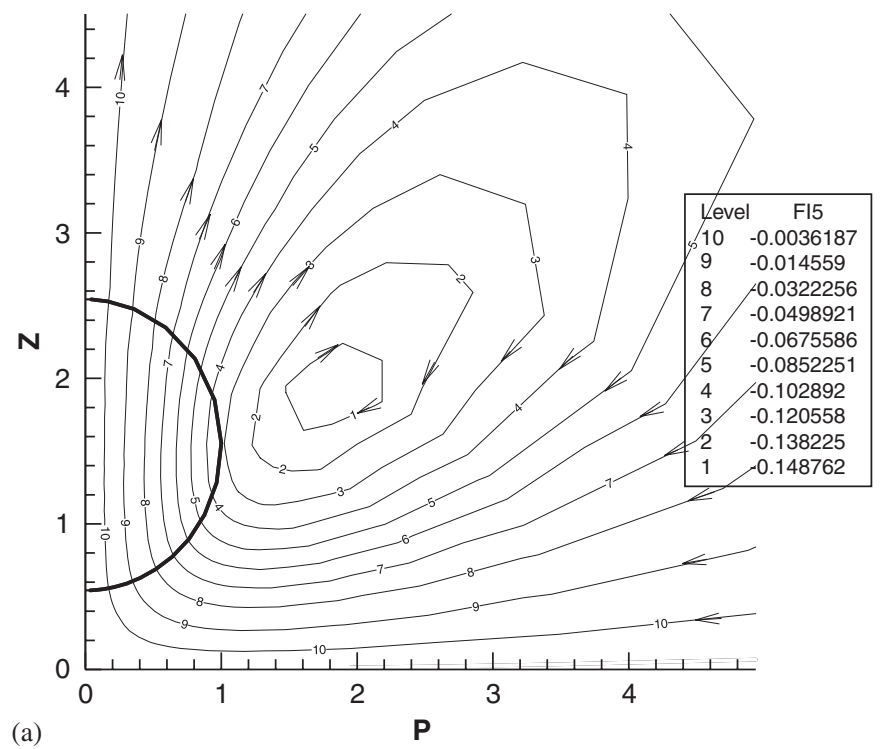

(a)

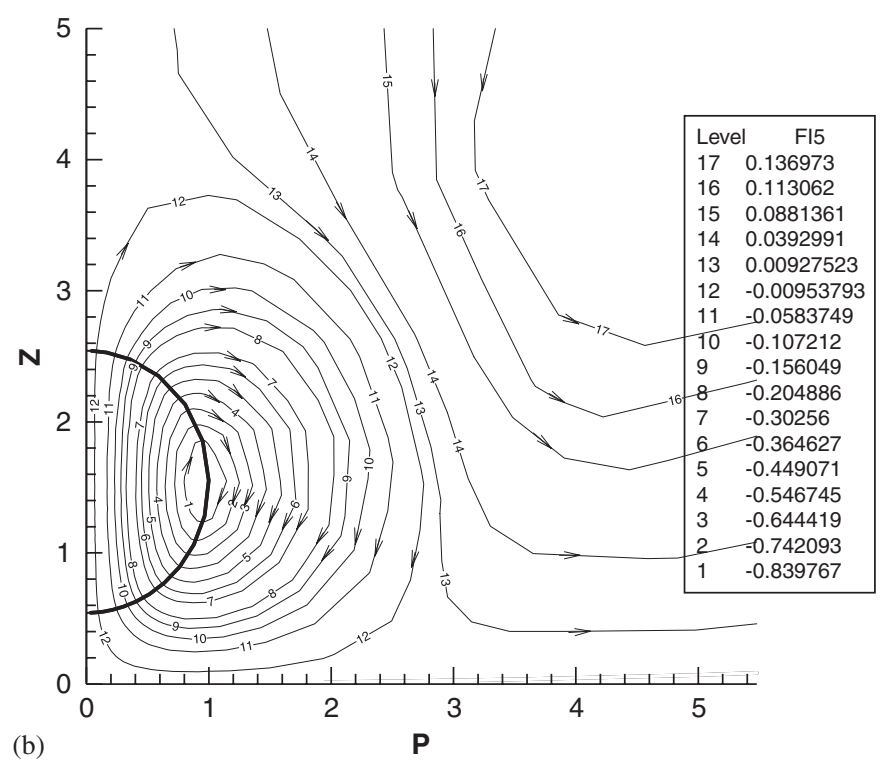

Fig. 4. Contours of stream function for the case of Fig. 3.

figures. Note that as $\sigma \rightarrow \infty$, the behavior of a drop approaches to that of a rigid sphere, and as $\sigma \rightarrow 0$ the behavior of the drop approaches to that of a bubble. Figs. 5 and 6 indicate that the mobility of a drop decreases with the increase in $\sigma$, which is expected since the larger the value of $\sigma$, the greater the viscous drag acting on a drop, as is illustrated in Figs. 7 and 8, where the drag on a rigid particle is based on the result of Wacholder and Weihs (1972). The negative value of the scaled drag force in these figures implies that its direction is always opposite to that of the movement of a drop, that is, it is a retardation force. Figs. 5 and 6 suggest that a drop can be treated as a rigid sphere if $\sigma$ exceeds about 100, and as a bubble if $\sigma$ is smaller than about 0.01 . In general, the larger the value of $\kappa a$, the larger is the mobility of a drop. A comparison between Figs. 6 and 8 reveals that for a fixed value of $\kappa a$ the qualitative behavior of the mobility is the same as that of the drag. According to Eq. (12), if $k a$ is very small, the present electrophoresis problem is essentially

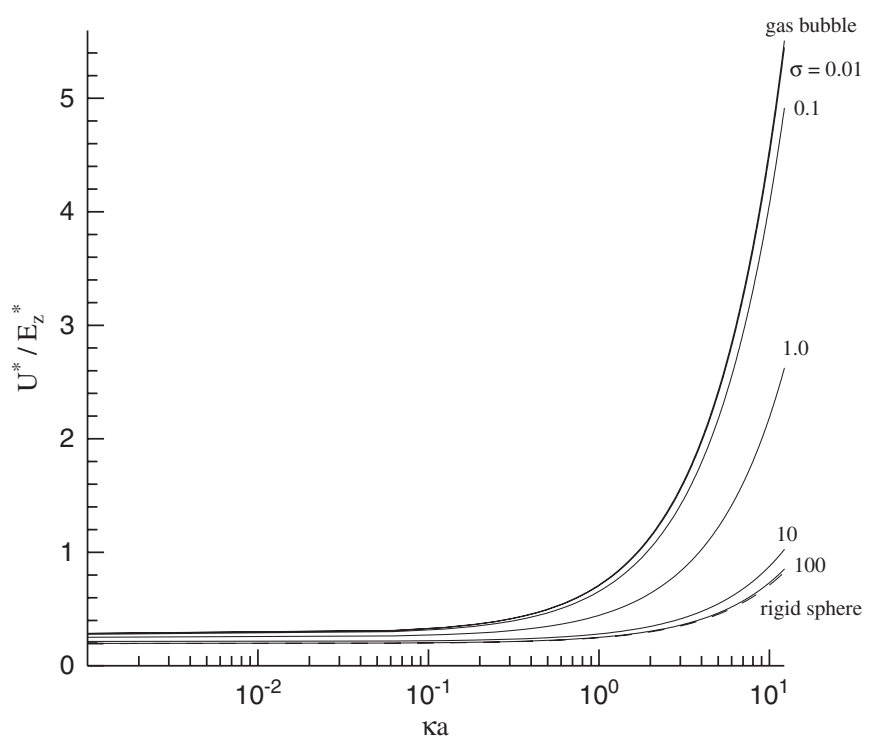

Fig. 5. Variation of scaled electrophoretic mobility $\left(U^{*} / E^{*}\right)$ as a function of $\kappa a$ for various values of $\sigma$ at $\eta_{0}=1.0$, dashed line represents result for a rigid sphere (Ennis and Anderson, 1997; Tang et al., 2001).

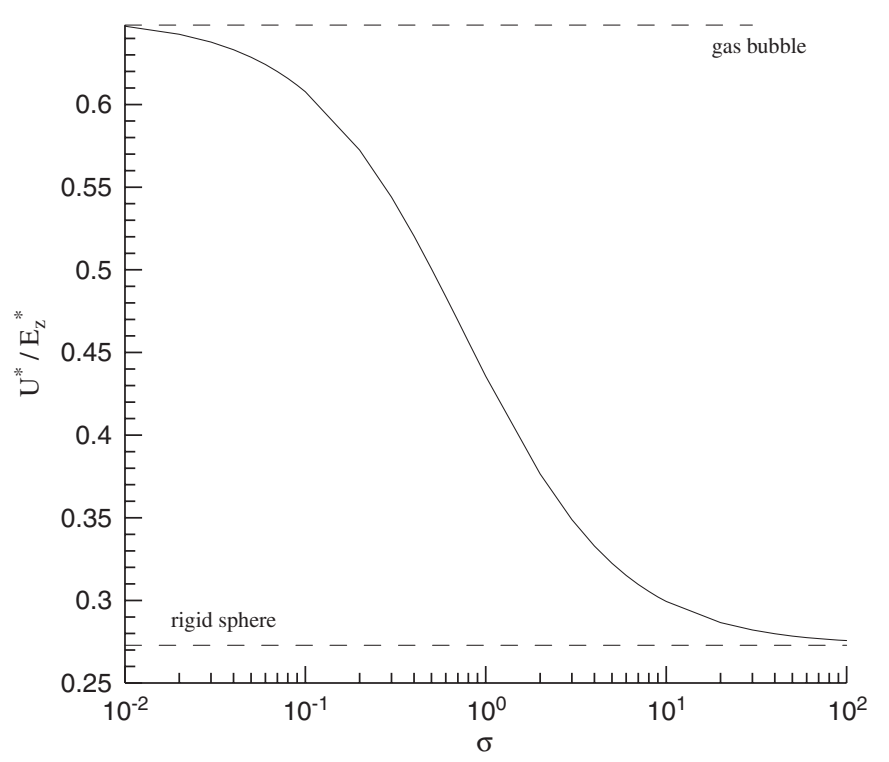

Fig. 6. Variation of scaled electrophoretic mobility $\left(U^{*} / E^{*}\right)$ as a function of $\sigma$ for the case when $\eta_{0}=1.0$ and $\kappa a=1.0$, dashed line represents result for a rigid sphere (Ennis and Anderson, 1997; Tang et al., 2001).

the same as a pure hydrodynamic problem. In this case, the mobility of a drop depends mainly upon the viscosity ratio $\sigma$. For instance, if $\kappa a=0.01$, the mobility of rigid sphere/mobility of drop with $\sigma=1.0$ is equal to 0.672 , which is about the same as the ratio (drag on rigid sphere/drag on drop). However, as $\kappa a$ increases, the ratio (mobility of rigid sphere/mobility of drop) declines; for example, it becomes 0.325 at $\kappa a=10$. In other words, the rate of increase in the mobility increases with the increase in $\kappa a$, as is seen in Fig. 5. As mentioned previously, this is because the larger the value of $\kappa a$, the greater the electric driving force acting on a drop. 


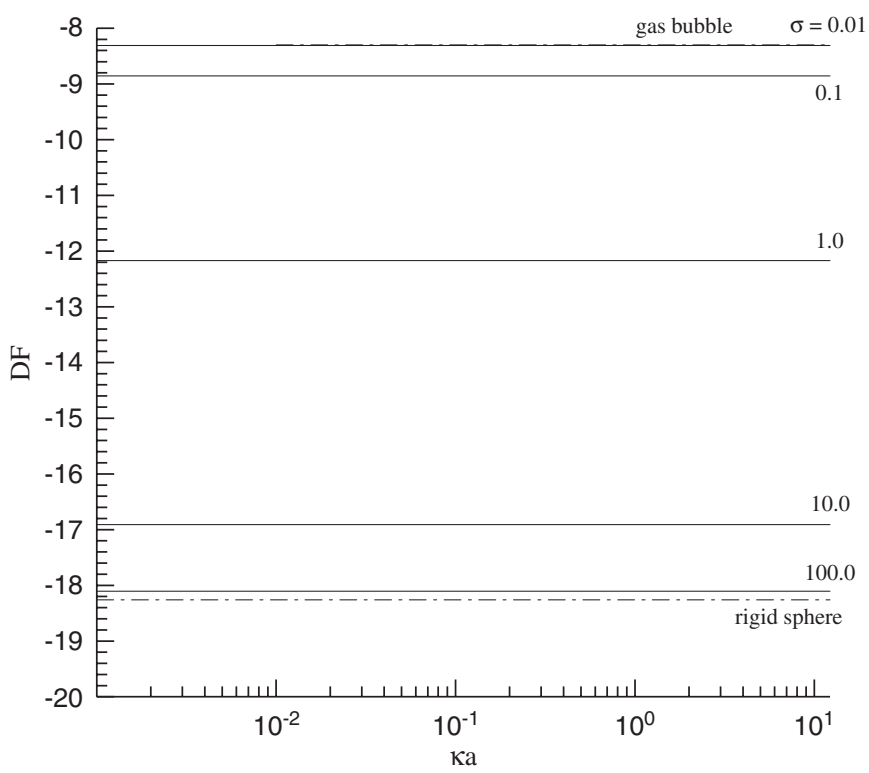

Fig. 7. Variation of scaled drag force DF as a function of $\kappa a$ for various values of $\sigma$ at $\eta_{0}=1.0$. Dash-dot line represents result for a rigid sphere (Wacholder and Weihs, 1972).

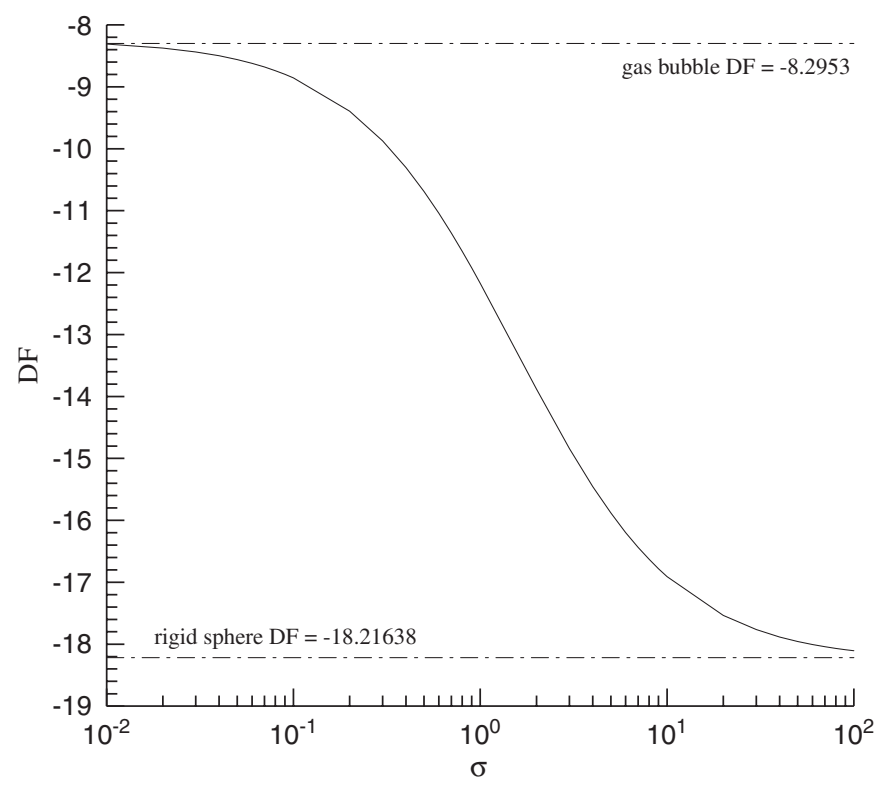

Fig. 8. Variation of scaled drag force DF on the drop surface as a function of $\sigma$ for the case when $\eta_{0}=1.0$ and $\kappa a=1.0$, dashed line represents result for a rigid sphere (Wacholder and Weihs, 1972).

The influence of the distance between drop and surface, measured by $\eta_{0}=\cosh ^{-1}(h / a)$, on the electrophoretic behavior of a drop is presented in Fig. 9. This figure reveals that for a fixed value of $\kappa a$ the mobility of a drop increases with its distance from a plane; similar behavior was also observed in the case of a rigid sphere (Ennis and Anderson, 1997; Tang et al., 2001). It is expected that as $\eta_{0} \rightarrow \infty$, the behavior of a drop approaches to that of an isolated drop in an infinite liquid. Note that if a drop is sufficiently close to a plane, its mobility may change

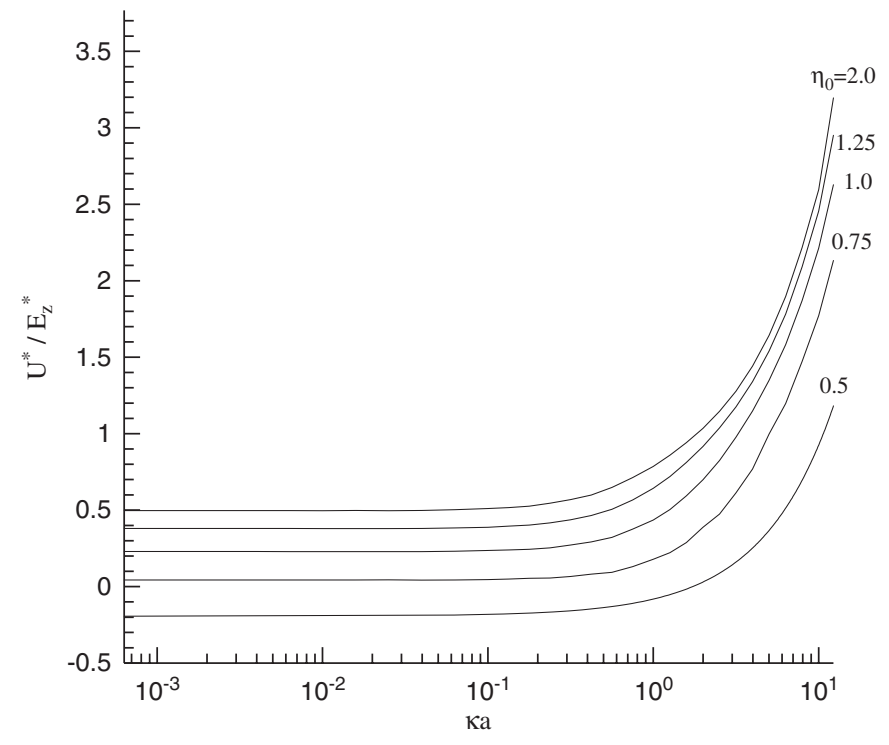

Fig. 9. Variation of scaled electrophoretic mobility $\left(U^{*} / E^{*}\right)$ as a function of $\kappa a$ for various values of $\eta_{0}$ at $\sigma=1.0$.

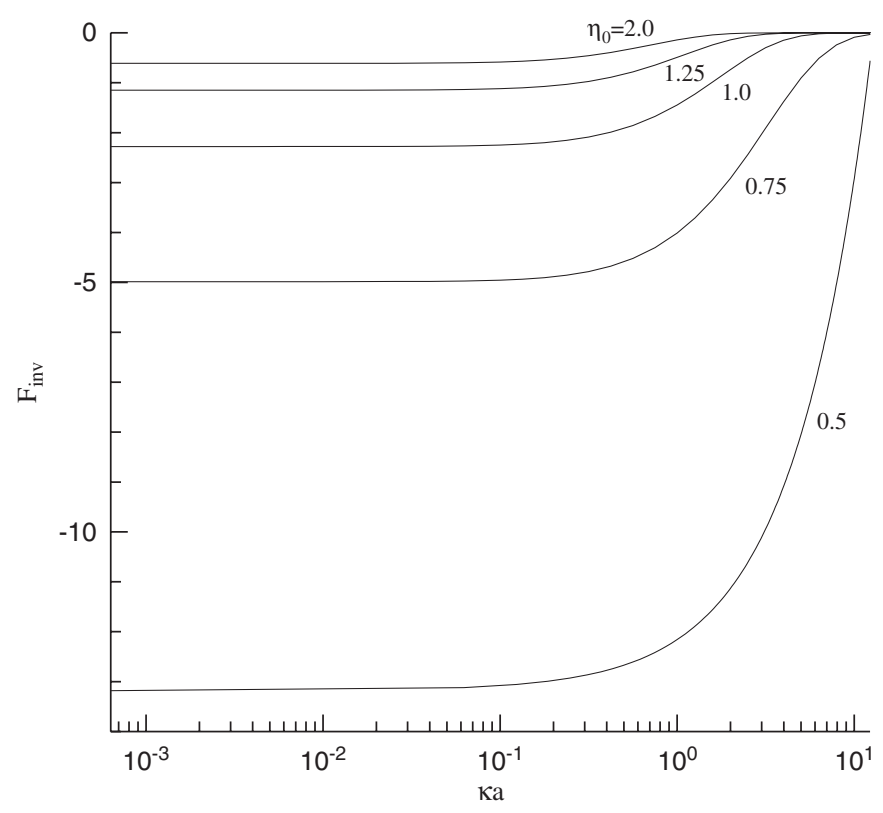

Fig. 10. Variation of scaled induced electric force $F_{\text {inv }}$ as a function of $\kappa a$ for various values of $\eta_{0}$ at $\sigma=1.0$.

sign, that is, it moves in the opposite direction as that of the applied electric field. This phenomenon was also observed in the case of a rigid sphere (Tang et al., 2001; Chih et al., 2002), and can be explained by the effect of the deformation of the double layer surrounding a drop. As illustrated in Fig. 3(a), if a drop is sufficiently close to a plane, the double layer surrounding the former will be distorted by the latter, that is, the equilibrium electric field constructed by the drop will be compressed by the plane. In this case, an electric force, which is in the opposite direction as that arises from the applied electric field, is induced. Fig. 10 illustrates the variation of the scaled 
magnitude of the electric force acting on a drop arising from this induced electric field, $F_{\text {inv }}$, as a function of $\kappa a$. This figure shows that $F_{\text {inv }}$ increases with the decrease in the distance between the drop and the plane. Note that if $\eta_{0}$ is smaller than about $0.5,\left|F_{\text {inv }}\right|$ becomes greater than the scaled electric force arising from the applied electric field, which is about 7.051 at $\eta_{0}=0.5$ for the present case.

Although we consider the case when only one plate is present, the result obtained is also applicable to the case when a drop is moving normal to one of the two parallel plates, if it can be considered to be sufficiently far away from one of those plates. The electrophoresis of droplet between two electrode surfaces is a typical example. The criterion for the critical distance can be inferred from the result of Wacholder and Weihs (1972). For a system consisting of a drop and a plate they concluded that if $\eta_{0} \geqslant 5.5$, or the ratio (drop-plate distance/drop radius) $\geqslant 122.348$, the drag on the drop is essentially the same as that when the plate is absent. The deformation of droplet is neglected in the present analysis. According to Taylor and Acrivos (1964), the deformation of a drop is proportional to $R e^{2}$, or to the Weber number $W e=\rho U^{2} a / \sigma_{1}, R e$ and $\sigma_{1}$ being, respectively, the Reynolds number and the surface tension of drop liquid. In our case, because typical value of $R e$ is on the order of $10^{-10}-10^{-8}$ and that of We is on the order of $10^{-17}$, the deformation of a drop can be neglected.

\section{Conclusions}

The boundary effect on the electrophoresis of a non-rigid particle is investigated by considering a non-conducting Newtonian drop moving normal to a plane. The present boundaryvalued problem is solved numerically by an orthogonal collocation method and the influences of the key parameters of the system under consideration are investigated through numerical simulation. We found that, in general, the thinner the double layer surrounding a drop and/or the longer its distance from a planar surface, the larger its mobility. Also, if a drop is sufficiently close to a plane, its mobility may change sign. These results are similar to the case of a rigid particle. The mobility of a drop decreases with the increase in the ratio (viscosity of drop fluid/viscosity of dispersion medium). Under the conditions assumed, a drop can be treated as a rigid sphere if the viscosity ratio exceeds about 100 , and as a bubble if it is smaller than about 0.01 .

\section{Notation}

\begin{tabular}{|c|c|}
\hline$a$ & radius of particle, $\mathrm{m}$ \\
\hline$c$ & focus length of bipolar coordination, $\mathrm{m}$ \\
\hline $\mathrm{DF}$ & hydrodynamic drag force, $\mathrm{N}$ \\
\hline$e$ & charge of electron, $1.6 \times 10^{-19} \mathrm{C}$ \\
\hline $\mathbf{E}_{\mathbf{z}}$ & applied electric field, $\mathrm{V} / \mathrm{m}$ \\
\hline$F_{\mathrm{inv}}$ & $\begin{array}{l}\text { electrical force induced by the imbalance charge } \\
\text { distribution, } \mathrm{N}\end{array}$ \\
\hline$F$ & force, $\mathrm{N}$ \\
\hline$h$ & distance between center of particle and plane, $\mathrm{m}$ \\
\hline
\end{tabular}

$\begin{array}{ll}k_{B} & \text { Boltzmann constant, } 1.38 \times 10^{-23} \mathrm{~J} / \mathrm{K} \\ n_{j} & \text { number density of electrolytes } j, \text { number } / \mathrm{m}^{3} \\ n_{j 0} & \text { bulk density of electrolytes } j, \text { number } / \mathrm{m}^{3} \\ p & \text { pressure, } \mathrm{N} / \mathrm{m}^{2} \\ R e & \text { Reynolds number, dimensionless } \\ T & \text { absolute temperature, } \mathrm{K} \\ U & \text { terminal velocity, } \mathrm{m} / \mathrm{s} \\ \mathbf{v} & \text { velocity vector, } \mathrm{m} / \mathrm{s} \\ W e & \text { Weber number, } W e=\rho U^{2} a / \sigma_{1}, \text { dimensionless } \\ x & x=\text { cosh } \eta-\cos \xi, \text { dimensionless } \\ y & y \text {-component of Cartesian coordinates, m } \\ z & z \text {-component of Cartesian coordinates } \\ z_{i} & \text { valence of ionic species } i, \text { dimensionless }\end{array}$

Greek letters

$\begin{array}{ll}\alpha & n_{10} / n_{20}, \text { dimensionless } \\ \varepsilon & \text { permittivity, C/V/m } \\ \zeta_{a} & \text { zeta potential, } \mathrm{V} \\ \eta & \eta \text {-component of bipolar coordinates, } \mathrm{m} \\ \eta_{0} & \text { position of the liquid drop, } \mathrm{m} \\ \kappa & \text { reciprocal Debye length, } 1 / \mathrm{m} \\ \mu & \text { viscosity of dispersion medium, } \mathrm{kg} / \mathrm{m} / \mathrm{s} \\ \xi & \xi \text {-component of bipolar coordinates, } \mathrm{m} \\ \rho & \text { space charge density in dispersion medium, } \\ & \mathrm{C} / \mathrm{m}^{3} \\ \sigma & \text { viscosity ratio } \mu^{(i)} / \mu^{(e)}, \text { dimensionless } \\ \sigma & \text { surface tension, } \mathrm{kg} / \mathrm{s}^{2} \\ \phi & \text { electrical potential, } \mathrm{V} \\ \varphi & \varphi \text {-component of bipolar coordinates, deg } \\ \psi & \text { stream function, } \mathrm{m}^{3} / \mathrm{s}\end{array}$

Superscripts

$\begin{array}{ll}* & \text { scaled symbols } \\ e & \text { exterior of a drop } \\ i & \text { interior of a drop }\end{array}$

Subscripts

e equilibrium properties

$\delta \quad$ perturbed properties

\section{Acknowledgment}

This work is supported by the National Science Council of the Republic of China.

\section{References}

Ashok, M., Joykrishna, D., 2005. Vesicles as pseudostationary phase for enantiomer separation by capillary electrophoresis. Journal of Chromatography A 1070, 185-192.

Baygents, J.C., Saville, D.A., 1991a. Electrophoresis of drops and bubbles. Journal of the Chemical Society-Faraday Transactions 87, 1883-1898.

Baygents, J.C., Saville, D.A., 1991b. Electrophoresis of small particles and fluid globules in weak electrolytes. Journal of Colloid and Interface Science 146, 9-37.

Booth, F., 1951. The cataphoresis of spherical fluid droplets in electrolytes. Journal of Chemical Physics 19, 1331-1342.

Breyer, E.D., Howard, S., Raje, N., Allison, S., Apkarian, R., Brown, W.V., Strasters, J.K., 2003. Study of lipid and apolipoprotein binding interactions 
using vesicle affinity capillary electrophoresis. Analytical Chemistry 75 5160-5169.

Chih, M.H., Lee, E., Hsu, J.P., 2002. Electrophoresis of a sphere normal to a plane at arbitrary electrical potential and double layer thickness. Journal of Colloid and Interface Science 248, 383-388.

Craxford, S.R., Gatty, O., McKay, H.A.C., 1937. The theory of electrocapillarity-Part VI. A note on electrophoresis. Philosophical Magazine 25, 172.

Ennis, J., Anderson, J.L., 1997. Boundary effects on electrophoretic motion of spherical particles for thick double layers and low zeta potential. Journal of Colloid and Interface Science 185, 497-514.

Finlayson, B.A., 1980. Nonlinear Analysis in Chemical Engineering. McGrawHill, New York.

Happel, J., Brenner, H., 1983. Low-Reynolds Number Hydrodynamics. Martinus Nijhoff, Dordrecht.

Keh, H.J., Anderson, J.L., 1985. Boundary effects on electrophoretic motion of colloidal spheres. Journal of Fluid Mechanism 153, 417-439.

Kelsall, G.H., Tang, S.Y., Yurdakul, S., Smith, A.L., 1996. Electrophoretic behaviour of bubbles in aqueous electrolytes. Journal of the Chemical Society-Faraday Transactions 2 92, 3887-3893.

Lee, E., Kao, J.D., Hsu, J.P., 2002. Electrophoresis of a nonrigid entity in a spherical cavity. Journal of Physical Chemistry B 106, 8790-8795.

Lee, E., Hu, J.K., Hsu, J.P., 2003a. Electrophoresis of concentrated mercury drops. Journal of Colloid and Interface Science 257, 250-257.

Lee, E., Fu, C.H., Hsu, J.P., 2003b. Electrophoresis of a concentrated dispersion of nonrigid particles. Langmuir 19, 3035-3040.

Lee, E., Tang, Y.P., Hsu, J.P., 2004. Electrophoresis of a membrane-coated sphere in a spherical cavity. Langmuir 20, 9415-9421.

Lee, E., Chang, C.J., Hsu, J.P., 2005a. Electrophoresis of a concentrated aqueous dispersion of non-Newtonian drops. Journal of Colloid and Interface Science 282, 486-492.
Lee, E., Huang, T.H., Hsu, J.P., 2005b. Sedimentation of a composite particle in a spherical cavity. Langmuir 21, 1729-1737.

Levich, V.G., 1962. Physicochemical Hydrodynamics. first ed. Prentice-Hall, New York. (Chapter IX).

Levine, S., O'Brien, R.N., 1973. Theory of electrophoresis of charged mercury drops in aqueous-electrolyte. Journal of Colloid and Interface Science 43, 616-629.

O'Brien, R.W., White, L.R., 1978. Electrophoretic mobility of a spherical colloidal particle. Journal of the Chemical Society-Faraday Transactions $274,1607-1626$.

Ohshima, H., 1997. A simple expression for the electrophoretic mobility of charged mercury drops. Journal of Colloid and Interface Science 189, 376 -378 .

Ohshima, H., 1999. Electrokinetic phenomena in a concentrated dispersion of mercury drops. Journal of Colloid and Interface Science 218, 533-544.

Ohshima, H., 2003. Electrophoretic mobility of a liquid drop in a salt-free medium. Journal of Colloid and Interface Science 263, 333-336.

Ohshima, H., Healy, T.W., White, L.R., 1984. Electrokinetic phenomena in a dilute suspension of charged mercury drops. Journal of the Chemical Society-Faraday Transactions 2 80, 1643-1667.

Tang, Y.P., Chih, M.H., Lee, E., Hsu, J.P., 2001. Electrophoretic motion of a charge-regulated sphere normal to a plane. Journal of Colloid and Interface Science 242, 121-126.

Taylor, T.D., Acrivos, A., 1964. On the deformation and drag of a falling viscous drop at low Reynolds number. Journal of Fluid Mechanics 18, $466-476$.

Wacholder, E., Weihs, D., 1972. Slow motion of a fluid sphere in the vicinity of another sphere or a plane boundary. Chemical Engineering Science 27, 1817-1828. 